\title{
BMJ Open Health-related quality Of Life In patients with advanced Soft TIssue sarcomas treated with Chemotherapy (The HOLISTIC study): protocol for an international observational cohort study
}

\author{
Eugenie Younger (D) , ${ }^{1,2}$ Robin L Jones (D) , ${ }^{1,3}$ Ingrid M E Desar, ${ }^{2}$ Clare Peckitt, ${ }^{1}$ \\ Winette T A van der Graaf, ${ }^{1,2,4}$ Olga Husson ${ }^{3,5}$
}

To cite: Younger E, Jones RL, Desar IME, et al. Health-related quality Of Life In patients with advanced Soft TIssue sarcomas treated with Chemotherapy (The HOLISTIC study): protocol for an international observational cohort study. BMJ Open 2020;10:e035171. doi:10.1136/ bmjopen-2019-035171

- Prepublication history for this paper is available online. To view these files, please visit the journal online (http://dx.doi. org/10.1136/bmjopen-2019035171).

Received 23 October 2019 Revised 11 February 2020 Accepted 01 May 2020

Check for updates

(C) Author(s) (or their employer(s)) 2020. Re-use permitted under CC BY-NC. No commercial re-use. See rights and permissions. Published by BMJ.

For numbered affiliations see end of article.

Correspondence to

Olga Husson;

olga.husson@icr.ac.uk

\section{ABSTRACT}

Introduction Chemotherapy is the mainstay of treatment for patients with advanced soft tissue sarcomas (STS). Treatment intent is usually palliative, aiming to improve symptoms, stabilise or reduce tumour burden and extend life. Clinical trials have traditionally used radiological response, time to progression and survival as measures of treatment efficacy. Health-related quality of life ( $\mathrm{HRQOL})$ is at least equally important or more important than survival for many patients with advanced cancer. Systematically collecting HRQoL data during chemotherapy can provide greater insight into treatment efficacy from the patient perspective.

The primary aims of this study are to evaluate HRQoL in patients with advanced STS treated with chemotherapy over time, explore the decision-making process and patient reflection post-treatment.

Methods and analysis This is an observational, international cohort study for 132 patients aged $\geq 18$ years with advanced STS treated at eight centres (three in the UK, five in the Netherlands). Patients will be recruited prior to starting first-line or third-line chemotherapy and invited to complete questionnaires using the Patient-Reported Outcomes Following Initial treatment and Long-term Evaluation of Survivorship registry (PROFILES); an established international registry for collection of cancer patient-reported outcomes. Online (or paper) questionnaires will be completed at baseline, each cycle of chemotherapy and 2-3 monthly during follow-up. The questionnaire package includes the Decisional Conflict Scale, Control Preferences Scale, Quality-Quantity Questionnaire, treatment expectations, European Organisation for the Research and Treatment of Cancer Quality of Life Questionnaire Core 30 (EORTCQLQ-C30), EORTC financial toxicity items, Work Ability Index, Functional Assessment of Cancer Therapy-General (FACT-G) items and Decisional Regret Scale. Clinical data will be extracted from patient records and linked with questionnaire responses. The primary outcome measure is the change in global HRQoL from baseline to after cycle 4 of first-line chemotherapy (based on published data showing that patients with advanced STS complete a median number of four cycles of first-line chemotherapy).

\section{Strengths and limitations of this study}

- Questionnaire data will be collected using the online Patient-Reported Outcomes Following Initial treatment and Long-term Evaluation of Survivorship system (PROFILES); a well-established electronic platform for the collection of patient-reported outcomes such as health-related quality of life (HRQLL).

- The longitudinal design of the study enables analysis of HRQoL across treatment lines.

- Although a validated HRQoL questionnaire specifically for patients with soft tissue sarcoma has not yet been developed, the study questionnaire package was developed in collaboration with patients and incorporates validated cancer-specific HRQoL questionnaires.

Ethics and dissemination Heath Research Authority and Research Ethics Committee (REC 17/N//0197). Results from the Health-related quality of Life In patients with advanced Soft TIssue sarcomas treated with Chemotherapy (HOLISTIC) study will be published in peerreviewed journals and disseminated at local, national and international conferences. We will also present our findings at any appropriate patient meetings and involve patients in study-related publications.

Trial registration number NCT03621332.

\section{BACKGROUND}

Soft tissue sarcomas (STS) are a group of rare and heterogeneous tumours, which account for approximately $1 \%$ of solid malignancies in adults. ${ }^{1}$ Due to the rarity and diverse presentation of STS, diagnosis can be delayed and around $10 \%$ of patients will be present with metastatic disease. ${ }^{2-5}$ Furthermore, many STS demonstrate an aggressive phenotype and approximately half of patients with intermediategrade or high grade localised tumours will develop metastatic disease after initial curative treatment. ${ }^{36}$ Despite advances 
in the treatment of many other cancers, the prognosis for patients with advanced, inoperable STS remains poor with a median overall survival of 12-19 months. ${ }^{78}$

Chemotherapy is the mainstay of treatment for patients with advanced STS. Other options include active surveillance for those with indolent or asymptomatic disease, radiotherapy or local therapies (eg, radiofrequency ablation or surgery) for patients with oligometastases, and best supportive care for those with end-stage disease and poor performance status (PS). ${ }^{6}$ The principal aims of chemotherapy are to ameliorate symptoms, slow or halt tumour growth and prolong survival. ${ }^{9}$ Standard first-line treatment, for the majority of STS subtypes, is anthracycline-based chemotherapy (usually doxorubicin), administered 3 weekly up to a maximum of six cycles due to the risk of cumulative cardiotoxicity. The combination of doxorubicin with ifosfamide is associated with higher response rates and longer progression-free survival, however with no improvement in overall survival and at the expense of increased toxicity. ${ }^{10}$ Doublet therapy may be considered for certain patients in whom a rapid response is clinically desirable, such as those with highly symptomatic chemosensitive disease. ${ }^{10}$ Other firstline chemotherapy regimens include weekly paclitaxel for patients with angiosarcoma and (rarely) low dose cyclophosphamide \pm prednisolone for frail, often elderly patients. ${ }^{11} 12$ A number of systemic agents are available following first-line anthracycline-based therapy, including trabectedin, pazopanib, gemcitabine \pm docetaxel, dacarbazine and eribulin.

Radiological response to first-line chemotherapy for advanced STS ranges from $10 \%$ to $50 \%$ (according to response evaluation criteria in solid tumours (RECIST V.1.1)), and is dependent on patient-specific-factors (eg, PS), tumour histology and chemotherapy regimen. ${ }^{6}$ Treatment decisions are often challenging due to modest response rates and potential treatment-related adverse side effects. For many patients with advanced cancer, health-related quality of life (HRQoL) is equally important or more important than survival when making treatment decisions. ${ }^{13} 14$ HRQoL is a multidimensional concept that represents the patient's perception of a disease and its treatment on physical, psychological and social aspects of their life. ${ }^{15}$ Systematically collecting HRQoL data over time may provide greater insight into treatment efficacy from the patient perspective and may enable a more detailed assessment of the risk-benefit ratio of chemotherapy for each individual patient. ${ }^{16}$

The efficacy of systemic therapies in patients with cancer has traditionally been evaluated with dimensional radiological response, progression-free survival and overall survival. The burden of symptoms in patients with advanced STS is high, particularly pain and dyspnoea. ${ }^{17}$ Despite the high symptom burden and palliative intent of treatment for most patients with advanced STS, the degree to which chemotherapy reduces symptoms of disease, improves or stabilises daily functioning and impacts HRQoL has rarely been measured or incorporated in the main endpoints of clinical trials. ${ }^{15}$ Collecting patientreported outcomes (PROs), such as HRQoL, is increasingly recognised to be key to fostering patient-centred care and influencing clinical decision-making. ${ }^{18}$ Patients' perspectives can also influence treatment decisions in an era of rising treatment costs and limited resources. Collection of PROs during chemotherapy has been well described. ${ }^{19}{ }^{20}$ Patient self-reporting improves symptom detection as clinicians frequently underestimate toxicities associated with systemic chemotherapy. ${ }^{20}{ }^{21}$ Recording electronic PROs in real time can also improve HRQoL, patient-provider communication, reduce hospitalisation and improve survival. ${ }^{22}$

Existing research into the HRQoL of patients with sarcoma has primarily focused on survivors of localised extremity STS, or specific subgroups of patients with advanced STS. ${ }^{23-26}$ For example, the SABINE study evaluated HRQoL among patients with metastatic STS or bone sarcoma who had attained a favourable response to chemotherapy. ${ }^{27}$ The PALETTE trial of pazopanib versus placebo, as second-line treatment (or greater) for patients with advanced STS, evaluated HRQoL as an exploratory endpoint. ${ }^{28}$ Although pazopanib did not improve HRQoL, meaningful improvement in progression-free survival was demonstrated with no associated impairment of HRQoL ${ }^{28}$ Hugdens et al analysed HRQoL for patients with advanced STS treated within the phase 3 trial of eribulin versus dacarbazine. ${ }^{29}$ This analysis demonstrated lower global health status and physical functioning scores, significantly worse loss of appetite, nausea and vomiting and insomnia in patients treated with dacarbazine on progression compared with patients treated with eribulin. ${ }^{29}$ The REGOSARC study of metastatic STS patients refractory to doxorubicin, posthoc quality-adjusted survival benefit analysis demonstrated superiority of regorafenib over placebo. ${ }^{30}$ The SARC021 phase 3 trial of doxorubicin versus doxorubicin plus evofosfamide reported no difference in HRQoL between treatment arms, despite a higher incidence of adverse events in the combination treatment arm. ${ }^{8}$ The phase 3 randomised study of trabectedin versus dacarbazine in patients with metastatic leiomyosarcoma or liposarcoma after failure of conventional chemotherapy used patientreported symptom scoring. ${ }^{31}$ A variety of HRQoL instruments were used to collect data in these studies as no specific HRQoL tool exists for STS-primarily due to the heterogeneity of this group of patients. ${ }^{16} 26$

In order for patients with advanced STS to make a wellinformed decision about chemotherapy and consider the possible effects on all aspects of their lives, clinicians should be able to provide HRQoL data. This will enhance the shared decision-making process between clinicians and their patients. To the best of our knowledge, this is the first study for patients with advanced STS which will evaluate HRQoL across chemotherapy treatment lines, consider the decision-making process (expectations and preferences for quantity of life vs QoL) and explore decisional regret. 


\section{METHODS/DESIGN}

The Health-related quality Of Life In patients with advanced Soft TIssue sarcomas treated with Chemotherapy (HOLISTIC) study is a longitudinal cohort questionnaire study for patients with advanced STS treated at eight sarcoma centres: three in the UK and five in the Netherlands (NL). The principal aim of this study is to assess how first-line chemotherapy affects global HRQoL over time (specified below) in patients with advanced STS. This study also aims to assess patient functioning (physical, psychological, emotional, social and role) and symptoms, before, during and after treatment with chemotherapy. The study will also explore treatment decision-making (expectations and preferences), financial toxicity of treatment and decisional regret after treatment. Detailed outcome measures are defined below.

\section{Patient and public involvement}

The study concept was developed in consultation with patient advocates, patients and their relatives, who felt that there was a lack of information on HRQoL in patients receiving chemotherapy for advanced soft tissue sarcoma in order to make a well-informed decision about treatment. Study documents were reviewed by the patients who are members of the Royal Marsden Hospital Patient and Public involvement panel. The panel provided feedback on the protocol, questionnaires, patient information sheet and informed consent form, with regard to content and readability. All suggestions were considered and changes incorporated in the final documents. In order to reduce time burden for patients, the questionnaire package was designed to minimise questionnaire fatigue. Patients will be involved in study-related presentations and publications.

\section{Eligibility criteria and materials \\ Inclusion criteria}

Eligible patients must be aged $\geq 18$ years with a diagnosis of advanced (not amenable to curative surgical resection) or metastatic STS, as confirmed by a sarcoma histopathologist, and has decided to start palliative chemotherapy following consultation with their oncologist. Patients must be enrolled prior starting first-line chemotherapy or third-line chemotherapy. Patients must be able to communicate in English or Dutch, and have mental capacity to provide informed consent and participate in the study (as determined by their treating physician). Patients must be able to complete questionnaires themselves, which is a prerequisite for patient-reported outcomes. Participants must be treated at one of the participating centres.

\section{Exclusion criteria}

Due to significantly different treatment protocols, patients with Ewing sarcoma, rhabdomyosarcoma, desmoplastic small round cell tumour and gastrointestinal stromal tumour are not eligible for the study.

\section{Data collection}

Data collection and online questionnaire administration will be done within the 'PROFILES' registry (PatientReported Outcomes Following Initial treatment and Long term Evaluation of Survivorship; www.profilesregistry.nl). The 'PROFILES' registry was established in the Netherlands (2009) for the study of the physical and psychosocial impact of cancer and its treatment in short-term and long-term cancer survivors. ${ }^{32}$ Since PROFILES has been established, studies have recruited over 30000 patients, resulting in more than 100 scientific publications. Security of the PROFILES server is established in accordance with current European norms (NEN-ISO/IEC 27002). Questionnaire data which are collected from participants (UK and NL) through the PROFILES registry is stored on a secure server in the Netherlands. This application has been developed to the requirements of the higher education and research community using end-to-end encryption.

\section{Recruitment}

Patients will be identified by a member of the sarcoma team who will check eligibility criteria using electronic patient records. Patients will be invited to participate in the outpatient clinic, provided with a patient information sheet and given the opportunity to ask any questions to a member of research team. Interested patients will be given the option to participate online or using paper versions of the questionnaire. Those who prefer online participation will receive an email which includes a link to the secure PROFILES website, unique username and password. The US Food and Drug Administration have made it clear that electronic capture of clinical trial source data is preferred over paper-based data collection. ${ }^{33}$ There is now widespread use of electronic patient reported outcome measures (PROMs) within clinical trials, and several reviews and meta-analyses have shown evidence of equivalence between electronic and paper administration of PROMs. ${ }^{345}$ In order to ensure that patients are not excluded if they are unable to use a computer, we have provided the option for paper questionnaire completion. Paper copies of the questionnaires will be entered using the data entry option of PROFILES, after which a quality control check will take place. This online data entry portal minimises the chance of errors as the answer options are selected from electronic lists. This also ensures that the paper questionnaire data can be extracted in the same format as the online questionnaire data. The PROFILES data manager will randomly choose five participants who have completed paper questionnaires and check the data entry for complete accuracy. If any errors are found, then all data will be rechecked and corrected where necessary.

All participants will complete an informed consent form. This can be done electronically (using PROFILES personal login details) or on paper if the patient prefers a hard copy. Patients are assured that non-participation has no consequences for their treatment or follow-up care. 
After informed consent, patients will be invited to complete the online (or paper) baseline questionnaire. This must be completed before starting first-line chemotherapy or third-line chemotherapy (third-line patient cohort). Patients can complete the online questionnaire on their own computer, tablet or mobile phone, or using a hospital computer if available. Patients who have completed the baseline questionnaire will then receive an email on day 1 of each cycle of chemotherapy, inviting them to complete a new questionnaire using their existing login details (usually every 3 weeks). Patients who prefer paper versions will be handed a hard copy of the questionnaire in the chemotherapy clinic, with the option to return this by mail using the prepaid envelopes provided, or hand to the research team in a sealed envelope. If chemotherapy is delayed for any reason, patients will be invited to complete the questionnaire when treatment has restarted. Baseline questionnaires will take around 20 minutes to complete and subsequent questionnaires will take around 10-15 minutes. Patients will complete a maximum of eight questionnaires during chemotherapy to reduce the potential for questionnaire fatigue. Patients have a 2-week period to complete each questionnaire and will be sent an electronic or paper reminder after 1 week if they have not completed a questionnaire.

When a participant stops first-line chemotherapy, or third-line chemotherapy (respective cohorts), for any reason (eg, all cycles completed, or disease progression), he/she will be invited to complete an 'end of chemotherapy' questionnaire. Patients will receive this invitation approximately 3 weeks after the last dose of chemotherapy. Thereafter, patients who have received first-line chemotherapy will be invited to complete 3 monthly follow-up questionnaires, and patients in the third-line chemotherapy cohort will complete 2 monthly follow-up questionnaires (due to the shorter prognosis). If a participant starts a new line of chemotherapy (eg, second-line chemotherapy) during the follow-up period, he/she will remain on the same follow-up schedule of questionnaires in order to follow the trajectory of their HRQoL during second-line treatment and beyond. Due to concern about questionnaire fatigue, it was felt that patients who had already completed 3-weekly questionnaires during first-line chemotherapy should remain on the 3 monthly follow-up schedule, rather than receiving questionnaires at the beginning of each cycle of secondline chemotherapy. Due to the time frame of the study, we will simultaneously recruit patients beginning thirdline treatment to explore HRQoL in patients with advanced STS who are further along their treatment trajectory.

Participants will be invited to complete follow-up questionnaires until he/she chooses to stop, is too unwell to continue or death occurs, whichever comes first, and up to a maximum of 2 years after study enrolment. Participants will only be enrolled once; either prior to firstline chemotherapy or before third-line chemotherapy. Throughout the study, it is the responsibility of the local sarcoma team to inform the study coordinator if a participant has died or is too unwell to continue.

\section{Case report forms}

After a patient has provided informed consent, clinical data will be collected from electronic patient records by a member of the research team. Data collection and storage will be maintained according to ICH-GCP (international good clinical practice) standards. Clinical details will be entered into the password protected database (MACRO) and stored using an anonymous patient identifier number. Personal identifiable clinical data of the patient will not leave the hospital where the patient is treated.

Case report forms (CRF) will be completed at four timepoints during the study. The first CRF should be completed after patient consent, and includes documentation of eligibility criteria, date of diagnosis of sarcoma, histological subtype and chemotherapy treatment regimen. The second part of the CRF should be completed when a patient stops first-line chemotherapy (or third-line chemotherapy for the third-line cohort). Information gathered at this timepoint includes reason for discontinuation of chemotherapy (eg, disease progression). The third point for CRF completion occurs when a patient stops participation in the study (eg, patient preference) and includes details of all chemotherapy regimens received during the study. The final point for CRF data collection is death notification, where applicable.

At the end of the study, questionnaire data will be linked with the clinical data contained within the CRF database using patient study numbers. Data linkage will be done by the study statistician at the Royal Marsden Hospital. Data will be recorded and retained in accordance with the Data Protection Act 1998.

\section{Questionnaires}

The baseline questionnaire contains questions on sociodemographic characteristics of the participant, such as marital and occupational status. Patients will also be asked a single screening question on health literacy which has validated among cancer patients and used in previous Dutch studies. ${ }^{36} 37$

The following internationally validated questionnaires and published questionnaires in studies of cancer patients will be used. These questionnaires have not been specifically validated in patients with advanced STS, however no STS-specific questionnaire has been developed to date. Permission to use all questionnaires has been obtained from authors. Formal licences are not required. Patients were involved in the design and review of the questionnaire package. All questionnaires were available in English. The Dutch questionnaire package was developed using validated Dutch versions of the questionnaires where possible or existing online Dutch translations of the questions. Where existing translations were not available, bilingual speakers assisted with formal 
foward-backward translation of questions and discrepancies resolved by $\mathrm{OH}$ (details below).

\section{EORTC-Quality of Life Questionnaire (EORTC-QLQ-C30)}

The European Organisation for the Research and Treatment of Cancer Quality of Life Questionnaire Core 30 (EORTC-QLQ-C30) V.3.0 was developed to assess QoL in patients with cancer. It has been translated and validated in over 100 languages, including English and Dutch. This questionnaire has 30 items, consisting of five functional scales (physical, role, cognitive, emotional and social), a global quality of life scale, three symptom scales (fatigue, pain, nausea and vomiting) and single items assessing common symptoms (dyspnoea, loss of appetite, sleep disturbance, constipation and diarrhoea) and perceived financial impact of the disease. ${ }^{38}$ After linear transformation, all scales and single-item measures range in score from 0 to 100 . A higher score on functional scales and global QoL means better functioning and HRQoL, whereas a higher score on the symptom scales means more complaints. ${ }^{38}$

\section{Financial toxicity}

Financial toxicity questions were selected from the item bank of the EORTC computer adaptive testing instrument and are validated in both English and Dutch languages. ${ }^{39}$ In combination with the EORTC-QLQ-C30 questionnaire, these questions will maximise information surrounding the financial impact of advanced STS and its treatment. This is particularly relevant for patients with rare cancers, such as STS, who may need to travel long distances to receive care at a specialist centre. ${ }^{40}$

\section{Functional Assessment of Cancer Therapy-General}

The Functional Assessment of Cancer Therapy-General (FACT-G) is a validated questionnaire which has been widely used to measure HRQoL in patients with cancer. The FACT-G has been translated and validated in many languages including English and Dutch. ${ }^{41}{ }^{42}$ Patients are asked to rate their response to several statements on a 5-point Likert scale from 'not at all' to 'very much'. ${ }^{41} \mathrm{We}$ have selected two items from the FACT-G questionnaire:'I am bothered by side effects of treatment' and 'I am able to enjoy life' as a summary measure of the burden of a given set of toxicities. ${ }^{43}$ Single items from the FACT-G questionnaire are not validated, however 'bother' from side effects has been shown to be associated with patients' ability to enjoy their lives. ${ }^{43}$

\section{Control Preferences Scale}

This validated questionnaire is designed to measure patients' preferred role in decision making versus their doctor's role. ${ }^{44}$ Patients are asked to choose from five options the phrase that best describes the role that they have taken in dealing with their cancer diagnosis and treatment decisions, and the role that they would have preferred (active/collaborative/passive role). ${ }^{44}$ Understanding patient preferences for control in treatment decision-making is crucial to improving shared decision-making and providing patient-centred care. ${ }^{45} 46$ Dutch translations of the Control Preferences Scale are available and have been used in previous studies in the PROFILES registry. ${ }^{47}$

\section{Decisional Conflict Scale}

The Decisional Conflict Scale measures personal perception of decision-making, and certainty or uncertainty over their choice. ${ }^{48}$ This includes determining the level of information and support to make the choice, whether the decision was in line with patient values and how satisfied they are/were with their decision. Items are given a score value of 0 (no) or 1 (yes). The total score can only be calculated if all four items are answered. The sum of the four items will range from 0 (extremely high decisional conflict) to 4 (no decisional conflict). A score of $\leq 3$ indicates decisional conflict. ${ }^{48}$ This four-item version of the Decisional Conflict Scale Questionnaire ('SURE') has been validated in English and a Dutch translation is available (psychometric properties have been partly confirmed in Dutch patients). ${ }^{49}$

\section{Quality-Quantity Questionnaire}

The Quality-Quantity Questionnaire (QQQ) tool is a validated construct consisting of eight questions which measure patient attitudes towards the trade-off of between quantity of life versus QoL (four questions for length of life (LOL) and four questions for QOL).$^{50} \mathrm{~A}$ separate score is calculated for LOL and QOL. The total score for all four questions (LOL or QOL) is calculated and the minimum score (4) is then subtracted. This answer is then divided by the range (20-4) to create a rescaling of 0-100. For example, if a person scores 3 (midpoint answer) for all four questions, the calculation would be: $(12-4) /(20-4)=0.50$ (midpoint overall score).$^{50}$ Decisionmaking in patients with advanced STS is extremely complex. Patient preferences for LOL and QOL are of utmost importance when weighing up the benefits and risks of treatments such as chemotherapy. Responses to these questions will allow insight into the preferences of patients with advanced STS and may help to inform shared treatment decisions. The original QQQ was written in the Dutch language (Professor Stiggelbout from the Netherlands) and has been translated and validated in English. ${ }^{50}$

\section{Expectations of treatment}

After the decision has been made to receive chemotherapy, patients are asked how likely they think that chemotherapy will improve survival, cure their cancer and improve symptoms due to cancer. ${ }^{51}$ Studies in patients with other metastatic solid tumours have shown that patients often overestimate their life expectancy and many believe that chemotherapy will be curative. ${ }^{51}$ Prognostic awareness has been associated with worse HRQoL in patients newly diagnosed with incurable (lung or GI) cancer. ${ }^{52}$ This question will be used to assess expectations of chemotherapy among advanced STS patients. These questions were originally written in English and therefore 
bilingual speakers performed a forward-backward translation of the questions into Dutch under the supervision of $\mathrm{OH}$.

\section{Work Ability Index}

The Work Ability Index (WAI) consists of questions which assess the ability to work, taking into account the demands of the work, health status and resources. ${ }^{53}$ There are seven questions from which we selected two general questions to inform future patients who are receiving chemotherapy on their potential ability to work during and after chemotherapy. These two questions are scored $0-10$ and $1-6$, respectively, and will be described in the analysis. ${ }^{53}$ The WAI was developed in Finland and is available in 24 languages, including English and Dutch. ${ }^{54}$ The validity and reliability of the WAI has been assessed in correlation analyses and used in many international research studies.

\section{Decisional Regret Scale}

Patients are asked to think about the decision they have made to receive chemotherapy and answer five statements on how strongly they agree/disagree: (1) it was the right decision, (2) I regret the choice that I made, (3) I would go for the same choice if I had to do it over again, (4) the choice did me a lot of harm and (5) the decision was a wise one. ${ }^{55}$ Items 2 and 4 should be reverse-coded so that, for each item, a higher number will indicate more regret. To help others interpret the score more readily with other scales ranging from 0 to 100 , these scores can then be converted to a $0-100$ scale by subtracting 1 from each item then multiply by $25 .^{55}$ To obtain a final score, the items are summed and averaged. A score of 0 means no regret; a score of 100 means high regret. ${ }^{55}$ The Decisional Regret Scale can be used to measure distress or regret after a healthcare decision such as the choice to receive chemotherapy. Regret about the decision to receive chemotherapy has not been previously measured in patients with advanced STS. The Decision Regret Scale is available in English and Dutch; Dutch translation by Leiden University Medical Center under supervision of Professor Dr Stiggelbout and Dr Pieterse, November $2010 .^{56}$

\section{Timepoints}

Questionnaire A is the baseline questionnaire, which should be completed before starting chemotherapy.

Patients are invited to complete Questionnaire B on day 1 of cycle 1 of first-line chemotherapy or third-line chemotherapy (third-line cohort). Patients are invited to complete Questionnaire C on day 1 of every cycle from Cycle 2 onwards and 3 weeks after the last cycle of chemotherapy. Questionnaire D is the follow-up questionnaire which is completed every 3 months after the end of chemotherapy for first-line chemotherapy patients, and every 2 months for the third-line chemotherapy patient cohort. Patients have a 2-week window to complete each questionnaire and will be sent a reminder after 1 week if they have not completed the questionnaire. Table 1 summarises enrolment, timepoints, the questions which are included in each questionnaire and the number of questions in each questionnaire.

\section{Endpoints}

The primary endpoint is change in EORTC-QLQ-C30 global HRQoL score (continuous scale) after treatment with first-line chemotherapy. Secondary endpoints are change in EORTC-QLQ-C30 physical, cognitive, social, role and emotional functioning scores (continuous) after treatment with first-line chemotherapy, and change in EORTC-QLQ-C30 symptoms (continuous) after treatment with first-line chemotherapy.

The study will also explore change in EORTC-QLQ-C30 global HRQoL score, EORTC-QLQ-C30 functioning scales and symptom scores after treatment with third-line chemotherapy (third-line patient cohort).

For all patients, we will examine whether there is an association between sociodemographic and clinical factors (including age, gender, relationship status, educational level, PS, tumour subtype, site of metastases, baseline anaemia $(\mathrm{Hb}<13 \mathrm{~g} / \mathrm{L}$ male, $<11.5 \mathrm{~g} / \mathrm{L}$ female $)$, lymphopoenia $<1 \times 10^{9} / \mathrm{L}$, lactate dehydrogenase (LDH) $>250 \mathrm{U} / \mathrm{L}$, hypoalbuminaemia $<35 \mathrm{~g} / \mathrm{L}$ ) and baseline EORTC-QLQ-C30 global HRQoL and/or change in physical, cognitive, social, role and emotional functioning during and after treatment. The study will evaluate whether there is an association between HRQoL and radiological response (according to RECIST V.1.1) to chemotherapy, and between HRQoL and financial toxicity.

The study will explore work ability in patients with advanced STS treated with chemotherapy, patient preferences for collaborative decision-making, decisional conflict about treatment, expectations of treatment with chemotherapy, preferences for quantity of life versus QoL and retrospective views on their decision to receive chemotherapy.

\section{Statistical analysis and power calculation}

Primary endpoint

Change in EORTC-QLQ-C30 global HRQoL score will be tested using a paired sample t-test from baseline to after four cycles with a two-sided 5\% significance level. For patients who do not complete four cycles, the last score/ observation (post baseline) will be carried forward for the analysis. Four cycles was chosen as the timepoint for the analysis based on a study of 488 patients with advanced STS treated at the Royal Marsden Hospital, showing that patients completed a median number of four cycles of chemotherapy (range 1-8)..$^{57}$

A sensitivity analysis will also be performed excluding those patients who do not reach four cycles.

If data are not normally distributed, then the Wilcoxon test will be used.

Secondary endpoints

Differences in physical, cognitive, social, role, emotional functioning and symptoms from baseline measurements 
Table 1 Enrolment and schedule of questionnaires

\begin{tabular}{|c|c|c|c|c|c|}
\hline Timepoint & Enrolment & Baseline & $\begin{array}{c}\text { Cycle } 1 \\
\text { chemotherapy }\end{array}$ & $\begin{array}{c}\text { Cycles 2-8, } \\
\text { and end of } \\
\text { chemotherapy }\end{array}$ & Follow-up \\
\hline Eligibility screen & $\mathrm{X}$ & & & & \\
\hline Informed consent & $X$ & & & & \\
\hline $\begin{array}{l}\text { QUESTIONNAIRES } \\
\text { (number of items) }\end{array}$ & & Questionnaire A & Questionnaire B & Questionnaire C & $\begin{array}{l}\text { Questionnaire } \\
\text { D }\end{array}$ \\
\hline Health literacy (1) & & $\mathrm{X}$ & & & \\
\hline Control Preferences Scale (2) & & $\mathrm{X}$ & & & \\
\hline Decisional Conflict Scale (4) & & $\mathrm{X}$ & & & \\
\hline Quality Quantity Questionnaire (8) & & $x$ & $x$ & $\mathrm{X}$ & \\
\hline $\begin{array}{l}\text { EORTC CAT items: financial } \\
\text { difficulties (5) }\end{array}$ & & $x$ & $x$ & $\mathrm{X}$ & $\mathrm{X}$ \\
\hline FACT-G items (2) & & - & $X^{*}$ & $\mathrm{X}$ & $\mathrm{X}$ \\
\hline Decisional Regret Scale (5) & & - & - & $\mathrm{X}$ & $\mathrm{X}$ \\
\hline Total number of questions & & 71 & 50 & 56 & 45 \\
\hline
\end{tabular}

*FACT-G, cycle 1 questionnaire includes the single statement 'I am able to enjoy life'. Cycles 2-8 and follow-up questionnaires include the additional statement 'I am bothered by the side effects of treatment'.

EORTC CAT, European Organisation for the Research and Treatment of Cancer computer adaptive testing; EORTC-QLQ-C30, European Organisation for the Research and Treatment of Cancer Quality of Life Questionnaire Core 30; FACT-G, Functional Assessment of Cancer Therapy-General.

over time will be analysed using mixed models, to allow for the repeated nature of the data by including subject as a repeated effect, and associations across visits (included as a fixed) will be investigated. The baseline score will also be fitted as a fixed effect in all models.

\section{Exploratory endpoints}

HRQoL of patients treated with third-line chemotherapy over time will be presented descriptively at each timepoint.

The association between sociodemographic and clinical factors (including age, gender, relationship status, educational level, PS, tumour subtype, site of metastases and baseline laboratory values), financial toxicity and radiological response with global HRQoL, physical, cognitive, social, role, emotional functioning and symptoms at each timepoint will be analysed using univariate mixed models as above. First time-invariant variables (eg, sex, race and age at diagnosis) will be assessed and then time-variant variables to evaluate whether time-specific conditions influence a linear trend of HRQoL over time. Interactions between factors and time will also be explored to assess whether trajectories of HRQoL over time differed by subgroups.

A multivariate model will be constructed as a function of time, sociodemographic and clinical characteristics, and interactions between time and other covariates. Any factors found significant $(\mathrm{p}<0.05)$ in univariate analysis will be tested in a multivariate mixed-effects models to see if factors of QOL are independent of each other. A backward selection method will be used $(p<0.05)$ to identify a parsimonious model.

Patient preference for treatment decision-making, decisional conflict, patient expectations, preference for quantity of life versus QoL, and retrospective views on their decision to receive chemotherapy will be presented descriptively with mean (SD) or median (IQR) at each timepoint as appropriate.

\section{Sample size}

For the primary endpoint, the difference from baseline to four cycles will be tested. A mean difference of 10 points in EORTC-QLQ-C30 global HRQoL score is deemed to be clinically relevant with an effect size of 0.3 , where the SD of the mean difference is $33.3 .{ }^{38}$ With a $90 \%$ power and a two-sided 5\% significance level, a total of 119 patients are required ${ }^{38}$ To allow for drop outs, an additional $10 \%$ of patients will be recruited, giving a total sample size of 132 .

We estimate that there will be approximately 30 patients in the third-line chemotherapy cohort within the timeframe of the study.

Missing data

Subjects who have completed online questionnaires will not have any missing data unless they have not completed 
the entire questionnaire. The electronic questionnaires have been programmed so that participants are not able to proceed to the next question until all of the questions on the current page have been answered. If patients have not completed the questionnaire, they will be sent an electronic reminder to finish the questionnaire within the time period specified. Regarding paper questionnaires, we will quantify the extent of missing items (ie, number of unanswered questions per patient) and describe these results in our findings. For the EORTC-QLQ-C30, we will follow the EORTC scoring manual guidelines for missing data and use imputation where appropriate. ${ }^{58}$ For all other questionnaires, scores cannot be calculated unless the patient has answered all of the items. We will categorise these responses as missing/not answered in the presentation of the results so that 'non-responses' are included in the analysis in order to minimise bias in interpretation.

\section{Author affiliations}

${ }^{1}$ Sarcoma Unit, Royal Marsden Hospital NHS Trust, London, UK

${ }^{2}$ Medical Oncology, Radboudumc, Nijmegen, Gelderland, The Netherlands

${ }^{3}$ Division of Clinical Studies, Institute of Cancer Research, London, UK

${ }^{4}$ Medical Oncology, Netherlands Cancer Institute-Antoni van Leeuwenhoek,

Amsterdam, Netherlands

${ }^{5}$ Department of Psychosocial Research and Epidemiology, Netherlands Cancer Institute, Amsterdam, Noord-Holland, The Netherlands

Acknowledgements We would like to thank the patients and their relatives for their philanthropic donations to the Sarcoma Research Unit at the Royal Marsden Hospital.

Contributors EY, RLJ, IMED, WTAvDG and OH were involved in the conceptualisation and design of the study. CP provided support for statistical methodology. EY was responsible for writing the protocol and obtaining ethical approval for the study. All authors read and approved the final manuscript.

Funding This research received no specific grant from any funding agency in the public, commercial or not-for-profit sectors. EY (clinical research fellow) is funded by the Sarcoma Research Unit at the Royal Marsden Hospital and was responsible for writing the protocol, and will be involved in the data collection, analysis, interpretation and writing of the manuscript as part of a $\mathrm{PhD}$ project. The joint sponsors for this study are the Institute of Cancer Research and the Royal Marsden NHS Foundation Trust. The sponsor contact is Dr Barbara Pittam(barbara.pittam@ icr.ac.uk). Role of sponsor: trial insurance and indemnity, scientific and statistical review of the protocol and study documents, ethical approval and protocol amendments, maintaining study master file, ensuring data and documentation are available for monitoring, inspection or audit, case report design, data analysis, preparation and dissemination of results (posters, presentations and publication) and data storage.

Competing interests RLJ is a consultant for: Adaptimmune, Blueprint, Clinigen, Eisai, Epizyme, Daichii, Deciphera, Immunedesign, Lilly, Merck, Pharmamar. WTAVDG has received research grants from Novartis and has been on advisory board from Bayer. The other authors declare that they have no competing interests.

Patient consent for publication Not required.

Ethics approval This study was approved by the Heath Research Authority and Research Ethics Committee of the UK (REC reference 17/NI/0197) and in the Netherlands at each participating centre (Radboud University Medical Centre and UMCG: 2018-4151, Erasmus Medical Centre: MEC-2018-1101, Leiden University Medical Centre: P18.179 P1a, Netherlands Cancer Institute: 2018-12-04 18.453) All participants will sign an informed consent form (electronic or paper version).

Provenance and peer review Not commissioned; externally peer reviewed.

Open access This is an open access article distributed in accordance with the Creative Commons Attribution Non Commercial (CC BY-NC 4.0) license, which permits others to distribute, remix, adapt, build upon this work non-commercially, and license their derivative works on different terms, provided the original work is properly cited, appropriate credit is given, any changes made indicated, and the use is non-commercial. See: http://creativecommons.org/licenses/by-nc/4.0/.

\section{ORCID iDs}

Eugenie Younger http://orcid.org/0000-0002-2088-3328

Robin L Jones http://orcid.org/0000-0003-4173-3844

\section{REFERENCES}

1 Burningham Z, Hashibe M, Spector L, et al. The epidemiology of sarcoma. Clin Sarcoma Res 2012;2:14.

2 Brouns F, Stas M, De Wever I. Delay in diagnosis of soft tissue sarcomas. Eur J Surg Oncol 2003;29:440-5.

3 Coindre JM, Terrier P, Guillou L, et al. Predictive value of grade for metastasis development in the main histologic types of adult soft tissue sarcomas: a study of 1240 patients from the French Federation of cancer centers sarcoma group. Cancer 2001;91:1914-26.

4 Ferguson PC, Deheshi BM, Chung P, et al. Soft tissue sarcoma presenting with metastatic disease: outcome with primary surgical resection. Cancer 2011;117:372-9.

5 Nijhuis PH, Schaapveld M, Otter R, et al. Epidemiological aspects of soft tissue sarcomas (STS)--consequences for the design of clinical STS trials. Eur J Cancer 1999;35:1705-10.

6 Dangoor A, Seddon B, Gerrand C, et al. Uk guidelines for the management of soft tissue sarcomas. Clin Sarcoma Res 2016;6:20.

7 Italiano A, Mathoulin-Pelissier S, Cesne AL, et al. Trends in survival for patients with metastatic soft-tissue sarcoma. Cancer 2011;117:1049-54.

8 Tap WD, Papai Z, Van Tine BA, et al. Doxorubicin plus evofosfamide versus doxorubicin alone in locally advanced, unresectable or metastatic soft-tissue sarcoma (th CR-406/SARC021): an international, multicentre, open-label, randomised phase 3 trial. Lancet Oncol 2017;18:1089-103.

9 Schöffski P, Cornillie J, Wozniak A, et al. Soft tissue sarcoma: an update on systemic treatment options for patients with advanced disease. Oncol Res Treat 2014;37:355-62.

10 Judson I, Verweij J, Gelderblom H, et al. Doxorubicin alone versus intensified doxorubicin plus ifosfamide for first-line treatment of advanced or metastatic soft-tissue sarcoma: a randomised controlled phase 3 trial. Lancet Oncol 2014;15:415-23.

11 Schlemmer M, Reichardt P, Verweij J, et al. Paclitaxel in patients with advanced angiosarcomas of soft tissue: a retrospective study of the EORTC soft tissue and bone sarcoma group. Eur J Cancer 2008;44:2433-6.

12 Mir O, Domont J, Cioffi A, et al. Feasibility of metronomic oral cyclophosphamide plus prednisolone in elderly patients with inoperable or metastatic soft tissue sarcoma. Eur J Cancer 2011;47:515-9.

13 Bottomley A. The cancer patient and quality of life. Oncologist 2002;7:120-5.

14 Shrestha A, Martin C, Burton M, et al. Quality of life versus length of life considerations in cancer patients: a systematic literature review. Psychooncology 2019;28:1367-80.

15 Kluetz PG, Slagle A, Papadopoulos EJ, et al. Focusing on core patient-reported outcomes in cancer clinical trials: symptomatic adverse events, physical function, and disease-related symptoms. Clin Cancer Res 2016;22:1553-8.

16 Younger E, Wilson R, van der Graaf WTA, et al. Health-Related quality of life in patients with sarcoma: enhancing personalized medicine. $J$ Clin Oncol 2018;36:1642-3.

17 Gough NJ, Smith C, Ross JR, et al. Symptom burden, survival and palliative care in advanced soft tissue sarcoma. Sarcoma 2011;2011:1-8.

18 Bottomley A, Pe M, Sloan J, et al. Analysing data from patientreported outcome and quality of life endpoints for cancer clinical trials: a start in setting international standards. Lancet Oncol 2016;17:e510-4.

19 Basch $\mathrm{E}$. The rationale for collecting patient-reported symptoms during routine chemotherapy. Am Soc Clin Oncol Educ Book 2014:161-5.

20 Di Maio M, Basch E, Bryce J, et al. Patient-Reported outcomes in the evaluation of toxicity of anticancer treatments. Nat Rev Clin Oncol 2016;13:319-25.

21 Basch E, Jia X, Heller G, et al. Adverse symptom event reporting by patients vs clinicians: relationships with clinical outcomes. J Natl Cancer Inst 2009;101:1624-32.

22 Basch E. Patient-Reported Outcomes - Harnessing Patients' Voices to Improve Clinical Care. N Engl J Med 2017;376:105-8. 
23 Davidson D, Barr RD, Riad S, et al. Health-Related quality of life following treatment for extremity soft tissue sarcoma. J Surg Oncol 2016;114:821-7.

24 Sachsenmaier SM, Ipach I, Kluba T. Quality of life, physical and mental status and Contentment of patients with localized soft tissue or bone sarcoma: a questionnaire analysis. Orthop Rev 2015;7:5920.

25 Fernandez-Pineda I, Hudson MM, Pappo AS, et al. Long-Term functional outcomes and quality of life in adult survivors of childhood extremity sarcomas: a report from the St. Jude lifetime cohort study. J Cancer Surviv 2017;11:1-12.

26 McDonough J, Eliott J, Neuhaus S, et al. Health-Related quality of life, psychosocial functioning, and unmet health needs in patients with sarcoma: a systematic review. Psychooncology 2019;28:653-64.

27 Reichardt P, Leahy M, Garcia Del Muro X, et al. Quality of life and utility in patients with metastatic soft tissue and bone sarcoma: the sarcoma treatment and burden of illness in North America and Europe (sabine) study. Sarcoma 2012;2012:740279

28 van der Graaf WTA, Blay J-Y, Chawla SP, et al. Pazopanib for metastatic soft-tissue sarcoma (PALETTE): a randomised, doubleblind, placebo-controlled phase 3 trial. Lancet 2012;379:1879-86.

29 Hudgens S, Forsythe A, Kontoudis I, et al. Evaluation of quality of life at progression in patients with soft tissue sarcoma. Sarcoma 2017;2017:2372135

30 Berry V, Basson L, Bogart E, et al. REGOSARC: regorafenib versus placebo in doxorubicin-refractory soft-tissue sarcoma-A qualityadjusted time without symptoms of progression or toxicity analysis. Cancer 2017;123:2294-302.

31 Demetri GD, von Mehren M, Jones RL, et al. Efficacy and safety of trabectedin or dacarbazine for metastatic liposarcoma or leiomyosarcoma after failure of conventional chemotherapy: results of a phase III randomized multicenter clinical trial. J Clin Oncol 2016;34:786-93.

32 van de Poll-Franse LV, Horevoorts N, van Eenbergen M, et al. The patient reported outcomes following initial treatment and long term evaluation of survivorship registry: scope, rationale and design of an infrastructure for the study of physical and psychosocial outcomes in cancer survivorship cohorts. Eur J Cancer 2011;47:2188-94.

33 Coons SJ, Eremenco S, Lundy JJ, et al. Pro) data electronically: the past, present, and promise of ePRO measurement in clinical trials. Patient 2015;8:301-9.

34 Gwaltney CJ, Shields AL, Shiffman S. Equivalence of electronic and paper-and-pencil administration of patient-reported outcome measures: a meta-analytic review. Value Health 2008;11:322-33.

35 Muehlhausen W, Doll H, Quadri N, et al. Equivalence of electronic and paper administration of patient-reported outcome measures: a systematic review and meta-analysis of studies conducted between 2007 and 2013. Health Qual Life Outcomes 2015;13:167.

36 Chew LD, Griffin JM, Partin MR, et al. Validation of screening questions for limited health literacy in a large Va outpatient population. J Gen Intern Med 2008;23:561-6.

37 Husson O, Mols F, Fransen MP, et al. Low subjective health literacy is associated with adverse health behaviors and worse health-related quality of life among colorectal cancer survivors: results from the profiles registry. Psychooncology 2015;24:478-86.

38 Cocks K, King MT, Velikova G, et al. Evidence-Based guidelines for determination of sample size and interpretation of the European organisation for the research and treatment of cancer quality of life questionnaire core 30. J Clin Oncol 2011;29:89-96.

39 EORTC Computerised Adaptive Tests (CAT). Item library: financial difficulties. Available: https://www.eortc.be/itemlibrary/ [Accessed 29 Aug 2019].
40 Paul CL, Hall AE, Carey ML, et al. Access to care and impacts of cancer on daily life: do they differ for metropolitan versus regional hematological cancer survivors? J Rural Health 2013;29 Suppl $1: s 43-50$.

41 Cella DF, Tulsky DS, Gray G, et al. The functional assessment of cancer therapy scale: development and validation of the general measure. J Clin Oncol 1993;11:570-9.

42 Bonomi AE, Cella DF, Hahn EA, et al. Multilingual translation of the functional assessment of cancer therapy (fact) quality of life measurement system. Qual Life Res 1996;5:309-20.

43 Pearman TP, Beaumont JL, Mroczek D, et al. Validity and usefulness of a single-item measure of patient-reported bother from side effects of cancer therapy. Cancer 2018;124:991-7.

44 Degner LF, Sloan JA, Venkatesh P. The control preferences scale. Can J Nurs Res 1997;29:21-43.

45 Singh JA, Sloan JA, Atherton PJ, et al. Preferred roles in treatment decision making among patients with cancer: a pooled analysis of studies using the control preferences scale. Am J Manag Care 2010;16:688-96.

46 Henrikson NB, Davison BJ, Berry DL. Measuring decisional control preferences in men newly diagnosed with prostate cancer. $J$ Psychosoc Oncol 2011;29:606-18.

47 Cuypers M, Lamers RED, de Vries M, et al. Prostate cancer survivors with a passive role preference in treatment decision-making are less satisfied with information received: results from the profiles registry. Urol Oncol 2016;34:482.e11-482.e18.

48 O'Connor AM. Decisional conflict scale. Available: http://decisionaid. ohri.ca/docs/develop/User_Manuals/UM_Decisional_Conflict.pdf [Accessed 29 Aug 2019].

49 Koedoot N, Molenaar S, Oosterveld P, et al. The decisional conflict scale: further validation in two samples of Dutch oncology patients. Patient Educ Couns 2001;45:187-93.

50 Stiggelbout AM, de Haes JC, Kiebert GM, et al. Tradeoffs between quality and quantity of life: development of the $\mathrm{QQ}$ questionnaire for cancer patient attitudes. Med Decis Making 1996;16:184-92.

51 Weeks JC, Catalano PJ, Cronin A, et al. Patients' expectations about effects of chemotherapy for advanced cancer. N Engl J Med 2012;367:1616-25

52 Nipp RD, Greer JA, El-Jawahri A, et al. Coping and prognostic awareness in patients with advanced cancer. J Clin Oncol 2017;35:2551-7.

53 Ilmarinen J. The work ability index (WAI). Occup Med 2007;57:160.

54 Gregorowitsch ML, van den Bongard HJGD, Couwenberg AM, et al. Self-Reported work ability in breast cancer survivors; a prospective cohort study in the Netherlands. Breast 2019;48:45-53.

55 O'Connor AM. Decisional regret scale. Available: http://decisionaid. ohri.ca/docs/develop/User_Manuals/UM_Regret_Scale.pdf [Accessed 29 Aug 2019].

56 Leiden University Medical Center. Dutch translation of decisional regret scale, 2010. Available: https://decisionaid.ohri.ca/docs/ develop/Tools/Regret_Scale_Dutch.pdf [Accessed 10 Feb 2020].

57 Karavasilis V, Seddon BM, Ashley S, et al. Significant clinical benefit of first-line palliative chemotherapy in advanced soft-tissue sarcoma: retrospective analysis and identification of prognostic factors in 488 patients. Cancer 2008;112:1585-91.

58 Fayers PM, Aaronson NK, et al, Bottomley A,on behalf of the EORTC Quality of Life Group. The EORTC QLQ-C30 scoring manual. 3rd Edn. Brussels: European Organisation for Research and Treatment of Cancer, 2001. 
Correction: Health-related quality of Life in patients with advanced soft TIssue sarcomas treated with Chemotherapy (The HOLISTIC study): protocol for an international observational cohort study

Younger E, Jones RL, Desar IME, et al. Health-related quality Of Life In patients with advanced Soft TIssue sarcomas treated with Chemotherapy (The HOLISTIC study): protocol for an international observational cohort study BMJ Open 2020;10:e035171. doi: 10.1136/bmjopen-2019-035171.

The article was previously published with an error.

The information'Winette T.A. van der Graaf and Olga Husson had joint contributions as last authors' was missing.

Open access This is an open access article distributed in accordance with the Creative Commons Attribution Non Commercial (CC BY-NC 4.0) license, which permits others to distribute, remix, adapt, build upon this work non-commercially, and license their derivative works on different terms, provided the original work is properly cited, appropriate credit is given, any changes made indicated, and the use is non-commercial. See: http://creativecommons.org/licenses/by-nc/4.0/.

(C) Author(s) (or their employer(s)) 2020. Re-use permitted under CC BY-NC. No commercial re-use. See rights and permissions. Published by BMJ.

BMJ Open 2020;10:e035171corr1. doi:10.1136/bmjopen-2019-035171corr1

Check for updates 